\title{
A prosocial online game for social cognition training in adolescents with high-functioning autism: an fMRI study
}

This article was published in the following Dove Press journal:

Neuropsychiatric Disease and Treatment

17 March 2016

Number of times this article has been viewed

\author{
Un-sun Chung' \\ Doug Hyun Han ${ }^{2}$ \\ Yee Jin $\mathrm{Shin}^{3}$ \\ Perry F Renshaw ${ }^{4}$ \\ 'Department of Psychiatry, Kyungpook \\ National University Hospital, Daegu, \\ Republic of Korea; ${ }^{2}$ Department of \\ Psychiatry, Chung Ang University \\ Hospital, Seoul, Republic of Korea; \\ ${ }^{3}$ Department of Psychiatry, Yonsei \\ University Hospital, Seoul, Republic \\ of Korea; ${ }^{4}$ Brain Institute, Utah \\ University, Salt Lake City, UT, USA
} Department of Psychiatry, Chung Ang University Hospital, HeukSeok Dong, Dong Jack Gu, Seoul 156-755, Republic of Korea

Tel +82 262993132

Email hduk70@gmail.com
Correspondence: Doug Hyun Han

Abstract: To help patients with autism spectrum disorder (ASD) improve their social skills, effective interventions and new treatment modalities are necessary. We hypothesized that a prosocial online game would improve social cognition in ASD adolescents, as assessed using metrics of social communication, facial recognition, and emotional words. Ten ASD adolescents underwent cognitive behavior therapy (CBT) using a prosocial online game (game-CBT), and ten ASD adolescents participated in an offline-CBT. At baseline and 6 weeks later, social communication quality, correct identification of emotional words and facial emoticons, and brain activity were assessed in both groups. Social communication quality and correct response rate of emotional words and facial emoticons improved in both groups over the course of the intervention, and there were no significant differences between groups. In response to the emotional words, the brain activity within the temporal and parietal cortices increased in the game-CBT group, while the brain activity within cingulate and parietal cortices increased in the offline-CBT group. In addition, ASD adolescents in the game-CBT group showed increased brain activity within the right cingulate gyrus, left medial frontal gyrus, left cerebellum, left fusiform gyrus, left insular cortex, and sublobar area in response to facial emoticons. A prosocial online game designed for CBT was as effective as offline-CBT in ASD adolescents. Participation in the game especially increased social arousal and aided ASD adolescents in recognizing emotion. The therapy also helped participants more accurately consider associated environments in response to facial emotional stimulation. However, the online CBT was less effective than the offline-CBT at evoking emotions in response to emotional words.

Keywords: autism, online games, sociality, cognitive behavior therapy, functional magnetic resonance image

\section{Introduction}

\section{Social cognition in adolescents with autism spectrum disorder}

A deficit in social cognitive abilities, including accurately integrating, interpreting, and responding to social cues is a critical impairment in patients with autism spectrum disorder (ASD). ${ }^{1}$ The deficit of social cognition in patients with ASD has been associated with an impairment of language and communication in early childhood..$^{2,3}$ Furthermore, these patients are faced with challenges in late adolescence and adulthood with regard to building interpersonal relationships, occupational functioning, and participating in the community. ${ }^{4}$ Patients with ASD with high functioning are on one side of the autism spectrum, and interventions and treatment modalities are currently being developed to help them overcome these challenges in the social community. 4,5

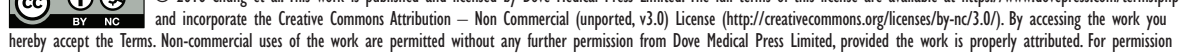
hereby accept the Terms. Non-commercial uses of the work are permitted without any further permission from Dove Medions.
for commercial use of this work, please see paragraphs 4.2 and 5 of our Terms (https://www.dovepress.com/terms.php). 
Currently, there are few evidence-based treatments or interventions for late adolescents or adult patients with highfunctioning autism. ${ }^{5}$

\section{Prosocial online game training for social cognition}

Previous studies have developed various programs to improve social skills and social cognition and their effectiveness was then quantified in terms of technique and platform. ${ }^{1,6,7}$ Several studies have simulated situations of a party or job interview ${ }^{8}$ and observed the frequency and style of personal interactions ${ }^{6}$ in ASD adolescents and ASD adults. Sociality in patients with ASD has been assessed by observing social performance and emotional responses. ${ }^{1,7,9-11}$ To this end, self-rated questionnaires that assess empathy and acceptance of other people have also been supplied to ASD adults. ${ }^{6}$ In other studies, social performance has been recorded while patients with ASD engage in a social task. ${ }^{1,7}$ Often, the emotional response is assessed by determining participants' accuracy with regard to emotion recognition in faces or voices or by matching expressions of emotion to emotional words. ${ }^{9,10}$ To characterize emotions and social cognition in patients with ASD in more detail, our previous study reported brain activity in response to emotional words and emoticons (animated face containing emotion) using functional magnetic resonance imaging (fMRI). ${ }^{11}$ Compared to healthy subjects, patients with ASD showed greater activation within the fusiform gyrus in response to the presentation of emotional words and facial emoticons. ${ }^{11}$

As a platform that provides opportunities to practice social interaction, virtual reality has been used to improve social training. ${ }^{12}$ In a study by Kandalaft et al, ${ }^{12}$ computerbased simulations of everyday life were presented on screen. After ten sessions of virtual reality social cognition training, eight ASD adolescents showed improvement on emotion recognition as well as social and occupational functions in a real-life situation. ${ }^{12}$ In another study, an interactive game system was thought to have possible therapeutic benefits in terms of memory, facial recognition, motor skills, and social interaction in ASD children. ${ }^{13}$

As another platform, video games have been suggested to improve sociality in patients with ASD. Similar to virtual reality therapy, ${ }^{12,14}$ online video games have merit to improve sociality in real-life experiences. These games provide a safe environment, repeated practice and exposure, naturalistic environments, various social scenarios, and replicated social conditions. ${ }^{15-18}$ Wilkinson et $\mathrm{al}^{18}$ summarize the benefits of online video games in supplementing therapeutic interactions for amelioration of anxiety, attention deficit hyperactivity disorder, and ASD; however, they should not replace therapists. Whalen et $\mathrm{al}^{15}$ developed a computer-assisted instructional program, Teach Town, which has a game-like structure for improving receptive language, social understanding, self-help, attention, memory, auditory processing, and academic skills for ASD children. Recent studies have reported that prosocial video game playing increases prosocial behaviors and thoughts in healthy adults. ${ }^{19,20}$ In addition, Greitemeyer et $\mathrm{al}^{21}$ showed that playing prosocial video games was associated with increased empathy and decreased reported pleasure at another's misfortune compared to neutral games. However, despite the growing recognition that virtual reality and video games can have therapeutic benefits, there has been little documented evidence and few studies assessing the treatment dynamics with the medium of online games.

\section{Hypothesis}

On the basis of previous studies, we assessed social cognition by having participants recognize emotional words and facial emoticons and by observing brain activity. We hypothesized that cognitive behavior therapy (CBT) using a prosocial online game would improve social cognition assessed using measures of social communication, facial recognition, and emotional words in ASD adolescents. In addition, we anticipated that improvements in social cognition in response to a prosocial game would be associated with brain activity within the fusiform gyrus in response to emoticons.

\section{Methods}

\section{Participants}

The Department of Psychiatry at Chung Ang University Hospital in Seoul, Republic of Korea, advertised the study of brain activity in response to emotional words and facial emoticons as well as CBT for sociality improvement in two phases. First, 15 ASD adolescent and age- and sex-matched healthy comparison subjects were recruited for the study of brain activity in response to emotional words and facial emoticons. Among those 15 ASD adolescents, 10 agreed to participate in the prosocial online game-CBT and the brain study. Second, 15 different adolescents with ASD were recruited to compare the effects of a prosocial online game (game-CBT) with offline-CBT. Brain activity in response to emotional words and facial emoticons for the 15 ASD adolescents in the first phase were analyzed and reported in a previous report. ${ }^{11}$ Inclusion criteria for the participants were 1) between the ages of 13 and 18 years, 2) diagnosed with 
ASD, 3) having an intelligence quotient (IQ) $\geq 70$, and 4) having an Autism Diagnostic Observation Schedule (ADOS) score in the range of 4-7. ${ }^{22}$ Exclusion criteria were as follows: 1) comorbid Axis I Disorders, as determined by the Korean Kiddie Schedule for Affective Disorders and SchizophreniaPresent and Lifetime version (K-SADS-PL) ${ }^{23}$ or psychiatry medication history within the last month; 2) history of head trauma with loss of consciousness, seizure disorder, multiple sclerosis, brain tumor, claustrophobia, metal implantation, or cerebrovascular accident; 3) serious or chronic medical illness; 4) IQ $<70$; and/or 5) a history of substance abuse. Twenty-five ASD adolescents were randomly assigned to the game-CBT $(\mathrm{N}=13)$ and offline-CBT groups $(\mathrm{N}=12)$. Of the 25 ASD adolescents, two ASD adolescents (one in gameCBT group and one in the offline-CBT group) were excluded because their IQ score was $<70$, one ASD adolescent in the game-CBT group was excluded because of attention deficit hyperactivity disorder, and one ASD adolescent in the gameCBT group was excluded because (s)he had been taking antipsychotic medication to reduce aggression. One ASD adolescent in the offline-CBT group dropped out due to lack of interest. Finally, ten ASD adolescents in the game-CBT group and ten ASD adolescents in the offline-CBT group completed all sessions of CBT. The research protocol was approved by the Chung Ang University Hospital Institutional Review Board. Written informed consent was provided by the ASD adolescents, and written informed consent was also provided by their parents.

\section{Procedures and assessment}

\section{Prosocial online game-CBT and offline-CBT}

The ASD adolescents in the game-CBT group were asked to sit in a closed room, log onto the game website, and play the online prosocial game, "Poki-Poki (http://www. pokipoki.co.kr/)", for $1 \mathrm{~h} / \mathrm{d}, 3$ times/wk with study personnel (two psychiatric social workers and one psychologist) for 6 weeks. There was no further assessment following the 6-week period of study. While playing the game, adolescents could engage in social activity by chatting, presenting virtual gifts, and imitating the other players to improve their own avatar and become friends with other avatars. During Poki-Poki game play, the study personnel conducted CBT by chatting with ASD adolescents in line with an established protocol (Table 1). To this end, participants were exposed to social and emotional words that were selected from a list of Korean emotional terms.${ }^{24}$ Similarly, ASD adolescents in the offline-CBT group were asked to participate in offline-CBT for $1 \mathrm{~h} / \mathrm{d}, 3$ times/wk with study personnel (two psychiatry social workers and one psychologist) for 6 weeks. During the 6-week offline-CBT, the study personnel conducted offlineCBT using same protocols as well as the same social and emotional words that were used in the online game-CBT. The protocol for social cognition consisted of 18 sessions (S) (two sessions for assessment and 16 sessions for CBT); S1: introduction; S2, 3: basic attitudes in conversation; S4, 5: body attitudes in conversation; S6, 7: verbal attitudes in conversation; S8: understanding what other people are saying; S9, 10: thought, emotion, and behavior; S11: voice; S12: self-assertiveness; S13: stress; S14, 15: coping strategies; and S16: review and future planning (Table 1).

\section{Assessment}

At baseline, all ASD adolescents in both groups were evaluated using K-SADS-PL, ADOS, the Social Communication Questionnaire Current form-Korean version (SCQ-K), an activity in which they identified emotional words and facial emoticons, and fMRI. After the last CBT session, all ASD adolescents in both groups repeated the SCQ-K, the activity where they identify emotional words and facial emoticons, and fMRI scanning. For confirmation of diagnosis, further evaluation with ADOS was assessed by a social worker (BGY), who has both clinical and research certificates for ADOS and Autism Diagnostic Interview, based on the training given by the supervisor Catherine Lord. The SCQ-K has been reported to have an inter-item consistency range from 0.84 to $0.93,25,26$ it has three subitems, including reciprocal social interaction (SCQ-I); communication (SCQ-C); and restricted, repetitive, and stereotyped pattern behavior (SCQ-B). The IQ scores and social communication in ASD adolescents were assessed with the Korean Wechsler Adult Intelligence Scale $^{27}$ and SCQ-K, ${ }^{26}$ respectively. Correct identification of emotional words ( 45 pleasant words +15 unpleasant words) and facial emoticons ( 45 pleasant faces +15 unpleasant faces) were assessed with 60 emotional words, with test-retest reliability of 0.82 , and 60 facial emoticons, with test-retest reliability of 0.84 , respectively. The explanation of emotional words and facial emoticons has been described in detail in our previous report. ${ }^{11}$

With the same sequences as our previous report, ${ }^{11}$ brain activity was assessed in two separate scans, in response to 1) 60 emotional words and 2) 60 facial emoticons. All information of brain activity was gathered from blood oxygen level dependent fMRI at $3 \mathrm{~T}$ (Achieva 3.0, Philips, Eindhoven, the Netherlands). For the fMRI protocol, 180 echo planar images ( 33 transverse slices, $4.0 \mathrm{~mm}$ thickness, voxel size of $1.8 \times 1.8 \times 4.0 \mathrm{~mm}, \mathrm{TE}=30 \mathrm{~ms}, \mathrm{TR}=3,000 \mathrm{~ms}$, 
Table I Outline of CBT for sociality

\begin{tabular}{ll}
\hline Session & Contents \\
\hline $\mathrm{I}$ & Introduction \\
& - ground rules and assessment of myself \\
& - introducing myself and others \\
& - setting goals \\
& Basic attitudes for conversation I \\
& - attitudes for conversation I \\
& - distance from other people I \\
& - mocial expressions during conversation I for conversation I
\end{tabular}

3

Basic attitudes for conversation 2

- attitudes for conversation 2

- distance from other people 2

- facial expressions during conversation 2

- mood for conversation 2

Body attitude for conversation I

- gazing at other's eye, nose, and face I

- gestures in conversation I

- listening courteously I

Body attitude for conversation 2

- gazing at other's eye, nose, and face 2

- gestures in conversation 2

- listening courteously 2

6

Verbal attitudes for conversation I

- speed of speech I

- rehearsal of speaking I

- topic transition I

7

Verbal attitudes for conversation 2

- speed of speech 2

- rehearsal of speaking 2

- topic transition 2

8

Understanding what others are saying

- five W's and one H

- tracing the order of what other people are saying

- tracing the order of events

Thought, emotion, and behavior I

- emotion expressed by body

- different emotions in different situation

- role playing during different situations

Thought, emotion, and behavior 2

- emotion expressed by body 2

- my expression and your expression

- "l" delivery and "You" delivery

Voice

- active voice

- passive voice

- aggressive voice

Self-assertiveness

- active, passive, and aggressive assertiveness

- confirming thoughts, emotion, and behavior

- my expression for assertiveness

Stress

- body responses to stress

- detecting my stress

- solutions to stress

Coping strategies I

- effective strategies for common situations

- steps for solving problems

- rehearsal

(Continued)
Table I (Continued)

\begin{tabular}{ll}
\hline Session & Contents \\
\hline I5 & Coping strategies 2 \\
& - effective strategies for individual situations \\
& - steps for solving individual problems \\
& - rehearsal \\
I6 & Review and future planning \\
& - review of sessions \\
& - award ceremony
\end{tabular}

Abbreviation: $\mathrm{CBT}$, cognitive behavior therapy.

flip angle $=90^{\circ}$, in-plane resolution $=128 \times 128$ pixels, field of view $[\mathrm{FOV}]=230 \times 230 \mathrm{~mm}$ ) were recorded at 3 -second intervals. For the anatomical imaging protocol, threedimensional $\mathrm{T}_{1}$-weighted magnetization-prepared rapid gradient echo data were gathered with these parameters: $\mathrm{TR}=2,000 \mathrm{~ms}, \mathrm{TE}=4 \mathrm{~ms}, \mathrm{FOV}=256 \times 256 \mathrm{~mm}, 340$ slices, $0.9 \times 0.9 \times 1.0 \mathrm{~mm}$ voxel size, flip angle $=30^{\circ}$. A video was presented for inducing brain activity that consisted of 450 second long, five continuous 90 -second segments. Each 90 -second segment consisted of three 30-second subsegments. A white cross on a black background (B), a single nonemotional word (or neutral face) (Match, M), and an emotional word (or facial emoticon) stimulation (Stimulation, S) were included in these 90 -second segments. The five segments were ordered as follows: B-M-S, B-S-M, M-B-S, S-B-M, and M-S-B. ${ }^{11}$

Brain activity data of individual adolescents were analyzed by a general linear model, with regressors defined to correspond to the time courses of the task conditions; each voxel of the time series was $z$-transformed. In addition, random effects analysis was applied to construct individual and group statistical parametric maps of brain activation. The group analysis consisted of a contrast between the averaged brain activation in response to (A) task (words or emoticons) and match at baseline versus (B) task and match at 6 weeks. The changes in brain activity in response to emotional words and emoticons between baseline and 6 weeks were compared using a paired $t$-test. These group maps were designed to correct multiple comparisons with a joint voxelwise significance and cluster size threshold to allow detection of weak but spatially extensive activations: the resulting false discovery rate was $<0.05$ for voxel sizes $>40 .^{28}$

\section{Statistical analysis}

Differences in demographic data, including age, IQ, SCQ scores, and percentage of correct answers in identifying emotional words/emoticons between the game-CBT 
group and the offline-CBT group were compared using the Mann-Whitney $U$-test and analysis of covariance (ANCOVA) test controlling for IQ. The changes in SCQ total, social interaction, communication, and stereotyped pattern behavior scores between two groups were compared using a repeated measure ANCOVA controlling for IQ. The changes in percentage of correct answers in identifying emotional words/emoticons between two groups were also compared using a repeated measure ANCOVA controlling for IQ.

\section{Results}

\section{Demographic characteristics}

There were no significant differences in sex, age, ADOS scores, Childhood Autism Rating Scale (CARS) scores, IQ, or SCQ scores between the game-CBT and the offline-CBT groups (Table 2).

\section{The change in social communication quality and correct identification of emotional words and emoticons}

In response to the 6-week CBT, SCQ-total scores were improved in both groups (game-CBT: $z=2.84, P<0.01$; offline-CBT: $z=11.1, P=0.04)$. However, there was no significant difference in the degree of improvement between the two groups $(F=0.17, P=0.69)$. In a post hoc test, the game-CBT group showed greater improvements in SCQ-I scores compared to the offline-CBT group $(F=9.40, P<0.01)$, while the offline-CBT group showed greater improvements in SCQ-B ( $F=6.23, P=0.02)$.

Compared with before CBT, the correct response rate of emotional words was improved in both groups after CBT (game-CBT: $z=2.67, P<0.01$; offline-CBT: $z=2.84$, $P<0.01)$. However, there was no significant difference in the degree of improvement between the two groups

Table 2 Demographic characteristics

\begin{tabular}{|c|c|c|c|}
\hline Variables & Game-CBT & Offline-CBT & Statistics \\
\hline Sex (male/female) & $(8 / 2)$ & $(9 / 1)$ & $\chi^{2}=0.39, P=0.53$ \\
\hline Age & $\mid 5.8 \pm 1.7$ & $16.3 \pm 1.5$ & $z=0.58, P=0.56$ \\
\hline \multicolumn{4}{|l|}{ ADOS } \\
\hline A & $3.4 \pm 0.7$ & $3.5 \pm 1.0$ & $z=0.04, P=0.96$ \\
\hline B & $6.6 \pm 3.2$ & $7.4 \pm 3.3$ & $z=0.49, P=0.62$ \\
\hline Diagnosis (autism/ASD) & $6 / 4$ & $5 / 5$ & $\chi^{2}=0.20, P=0.65$ \\
\hline CARS & $25.9 \pm 4.9$ & $27.5 \pm 5.6$ & $z=0.58, P=0.56$ \\
\hline IQ & $80.0 \pm 4.7$ & $80.4 \pm 8.0$ & $z=0.19, P=0.85$ \\
\hline \multicolumn{4}{|l|}{ SCQ } \\
\hline Total & & & $F=0.17, P=0.69^{a}$ \\
\hline Baseline & $20.5 \pm 4.2$ & $21.0 \pm 4.7$ & $z=0.45, P=0.65$ \\
\hline 6 weeks & $17.4 \pm 3.1$ & $18.3 \pm 3.3$ & $z=0.49, P=0.69$ \\
\hline SCQ-I & & & $F=9.40, P<0.0 I^{a, b}$ \\
\hline Baseline & $8.4 \pm 2.4$ & $8.0 \pm 2.3$ & $z=0.23, P=0.82$ \\
\hline 6 weeks & $6.3 \pm 1.3$ & $7.7 \pm 1.9$ & $z=1.81, P=0.06$ \\
\hline SCQ-C & & & $F=4.31, P=0.05^{a}$ \\
\hline Baseline & $7.2 \pm 2.3$ & $7.7 \pm 2.0$ & $z=0.57, P=0.57$ \\
\hline 6 weeks & $6.6 \pm 2.0$ & $5.8 \pm 1.3$ & $z=1.09, P=0.27$ \\
\hline SCQ-B & & & $F=6.23, P=0.02^{a, b}$ \\
\hline Baseline & $4.7 \pm 1.3$ & $5.5 \pm 1.4$ & $z=I .24, P=0.21$ \\
\hline 6 weeks & $4.9 \pm 1.4$ & $4.8 \pm 1.5$ & $z=0.23, P=0.8 \mathrm{I}$ \\
\hline \multirow[t]{3}{*}{ Emotional words (\%) } & & & $F=0.44, P=0.52^{\mathrm{a}}$ \\
\hline & $37.7 \pm 9.8$ & $39.0 \pm 11.6$ & $z=0.15, P=0.88$ \\
\hline & $43.7 \pm 11.7$ & $46.0 \pm 12.9$ & $z=0.19, P=0.84$ \\
\hline \multirow[t]{3}{*}{ Facial emoticons (\%) } & & & $F=2.72, P=0.12^{\mathrm{a}}$ \\
\hline & $51.0 \pm 11.4$ & $55.0 \pm 11.2$ & $z=0.75, P=0.44$ \\
\hline & $62.7 \pm 9.9$ & $63.3 \pm 11.4$ & $z=0.11, P=0.91$ \\
\hline
\end{tabular}

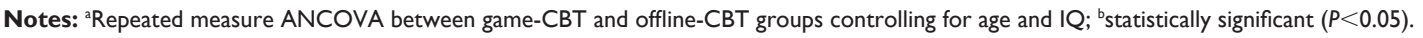

Abbreviations: CBT, cognitive behavior therapy; ADOS, Autism Diagnostic Observation Schedule; ASD, autism spectrum disorder; CARS, Childhood Autism Rating Scale; IQ, intelligence quotient; SCQ-I, social communication questionnaire current form-Korean version, reciprocal social interaction; SCQ-C, social communication questionnaire current form-Korean version, communication; SCQ-B, social communication questionnaire current form Korean version; restricted, repetitive, and stereotyped pattern behavior; ANCOVA, analysis of covariance. 
( $F=0.44, P=0.52$ ). Over the course of the intervention, the correct response rate to facial emoticons was improved in both groups (game-CBT: $z=2.85, P<0.01$; offline-CBT: $z=2.43, P=0.01)$. However, there was no significant difference in the degree of improvement between groups $(F=2.72, P=0.44)$.

\section{Brain changes in response to emotional words and facial emoticons}

After CBT, the game-CBT group showed that the brain activity in response to emotional words within the right temporal lobe, left superior parietal lobe, and left superior temporal lobe was increased compared to before CBT $(P<0.05)$. The offlineCBT group showed an increase in brain activity in response to emotional words within right cingulate gyrus and left parietal lobe in response to CBT $(P<0.05$; Figure 1, Table 3$)$.

CBT led to an increase in brain activity in response to facial emoticons within right cingulated gyrus, left medial frontal gyrus, left cerebellum, left fusiform gyrus, and left insular and sublobar area in the game-CBT group $(P<0.05)$. Similarly, the offline-CBT group showed an increase in brain activity in response to facial emoticons in the right anterior cingulate gyrus and left cingulate gyrus $(P<0.05)$ as well as a decrease of activity in the right medial frontal gyrus, left superior frontal gyrus, and left superior parietal lobe $(P<0.05$; Figure 1 , Table 3$)$. There were no brain regions showing decreased activity in response to emotional words and facial emoticons in the game-CBT group during the 6 weeks.

\section{Correlation between the changes in social communication, the correct identification of emotional words and emoticons, and brain activity}

In the game-CBT group, the changes in mean $\beta$ values within the left fusiform gyrus were positively correlated with the changes in the correct identification rate of emoticons at a trend level $(r=0.71, P=0.02)$ and negatively correlated with the changes in SCQ-I scores at a trend level $(r=-0.55$, $P=0.08$ ). There were no significant correlations between the changes in social communication, correct identification of emotional words and emoticons, and brain activity within other clusters.

\section{Discussion}

The current results suggest that game-CBT was as effective at improving sociality in ASD adolescents as offline-CBT. Moreover, game-CBT led to greater improvements in social interactions but fewer improvements in controlled, restricted, and stereotyped behavior in ASD adolescents compared to offlineCBT. In addition, patients in the game-CBT group exhibited different changes in blood flow in response to emotional words

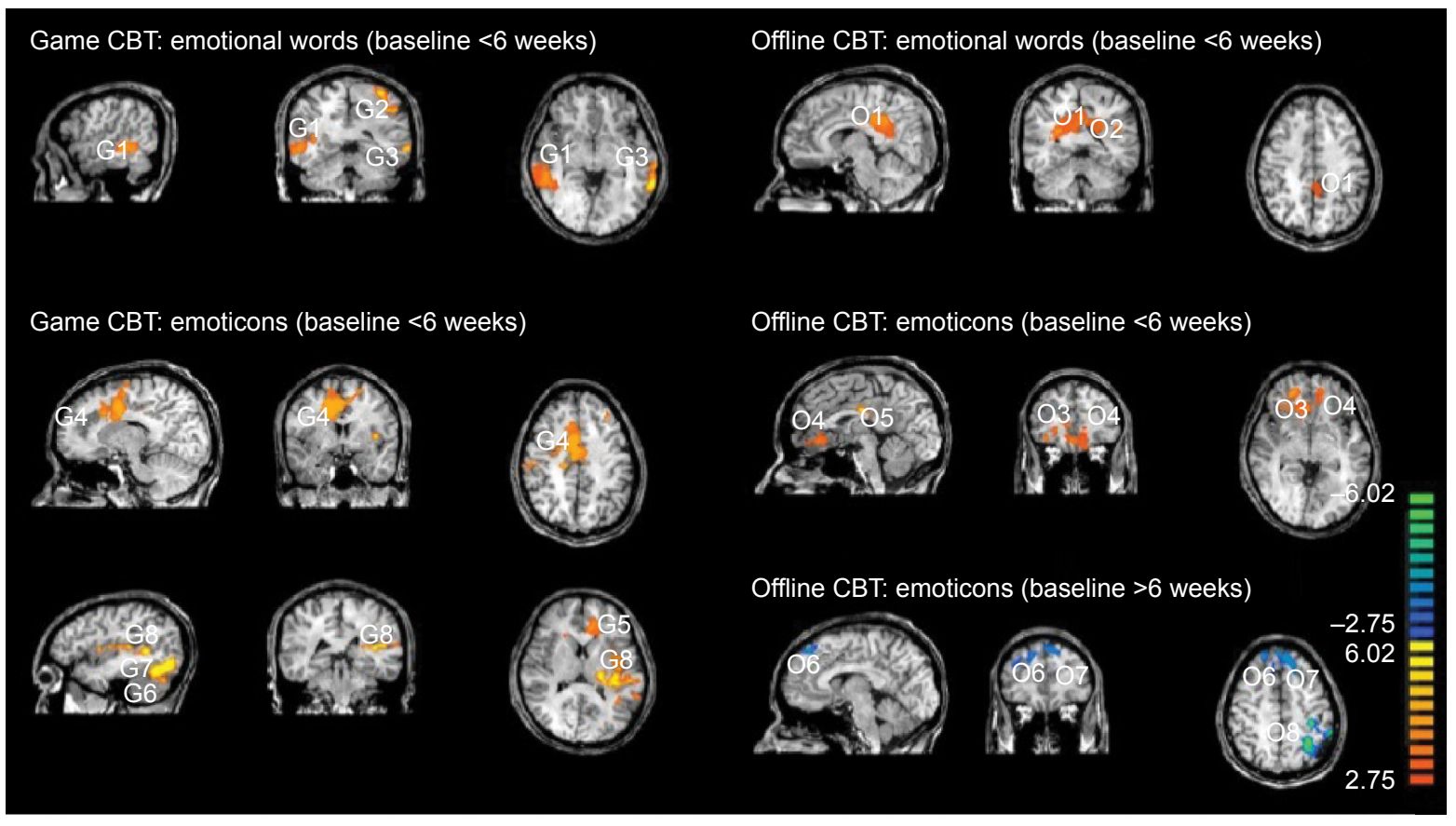

Figure I Changes in brain activity in response to emotional words and emoticon faces. Abbreviation: CBT, cognitive behavior therapy. 
Table 3 Changes in brain activity in response to emotional words and emoticon faces

\begin{tabular}{|c|c|c|c|c|c|}
\hline \multicolumn{3}{|c|}{ Talairach code } & \multirow[t]{2}{*}{ Voxels } & \multirow[t]{2}{*}{ FDR $<0.05$} & \multirow[t]{2}{*}{ Regions } \\
\hline $\mathbf{x}$ & $\mathbf{y}$ & $\mathbf{z}$ & & & \\
\hline \multicolumn{6}{|c|}{ Game-CBT: emotional words (baseline $<6$ weeks) } \\
\hline 50 & -39 & -3 & 210 & $P<0.0012$ & GI. Right temporal lobe BA 37 \\
\hline-32 & -46 & 51 & 191 & $P<0.0012$ & G2. Left superior parietal lobule, BA 7 \\
\hline-50 & -51 & 8 & 186 & $P<0.0012$ & G3. Left superior temporal gyrus, BA 39 \\
\hline \multicolumn{6}{|c|}{ Game-СВT: emotional words (baseline $>6$ weeks) } \\
\hline \multicolumn{6}{|c|}{ None } \\
\hline \multicolumn{6}{|c|}{ Game-CBT: facial emoticons (baseline $<6$ weeks) } \\
\hline II & 0 & 40 & 204 & $P<0.0017$ & G4. Right cingulate gyrus, BA 24 \\
\hline-21 & 39 & 22 & 194 & $P<0.0017$ & G5. Left medial frontal gyrus, BA 9 \\
\hline-19 & -64 & -19 & 182 & $P<0.0017$ & G6. Left cerebellum, posterior lobe \\
\hline-39 & -68 & -14 & 180 & $P<0.0017$ & G7. Left fusiform gyrus, BA 19 \\
\hline-39 & 6 & 9 & 177 & $P<0.0017$ & G8. Left insular and sublobar \\
\hline \multicolumn{6}{|c|}{ Game-CBT: facial emoticons (baseline $>6$ weeks) } \\
\hline \multicolumn{6}{|c|}{ None } \\
\hline \multicolumn{6}{|c|}{ Offline-CBT: emotional words (baseline $>6$ weeks) } \\
\hline 6 & -40 & 30 & 321 & $P<0.0009$ & OI. Right cingulate gyrus, BA 3I \\
\hline-42 & -30 & 24 & 292 & $P<0.0009$ & O2. Left parietal lobe white matter \\
\hline \multicolumn{6}{|c|}{ Offline-CBT: emotional words (baseline $>6$ weeks) } \\
\hline \multicolumn{6}{|c|}{ None } \\
\hline \multicolumn{6}{|c|}{ Offline-CBT: facial emoticons (baseline $<6$ weeks) } \\
\hline 19 & 42 & 2 & 115 & $P<0.0093$ & O3. Right anterior cingulate, BA 32 \\
\hline-2 & 34 & -2 & 83 & $P<0.0093$ & O4. Left anterior cingulate, BA 24 \\
\hline-1 & -33 & 26 & 81 & $P<0.0093$ & O5. Left cingulate gyrus, BA 23 \\
\hline \multicolumn{6}{|c|}{ Offline-CBT: facial emoticons (baseline $>6$ weeks) } \\
\hline 17 & 27 & 38 & 212 & $P<0.0021$ & O6. Right medial frontal gyrus, BA 6 \\
\hline-6 & 34 & 46 & 192 & $P<0.0021$ & O7. Left superior frontal gyrus, BA 8 \\
\hline-34 & -65 & 46 & 175 & $P<0.0021$ & O8. Left superior parietal lobe, BA 7 \\
\hline
\end{tabular}

Note: The cluster significance level of $P<0.05$ was estimated through Monte Carlo simulations using the BrainVoyager Cluster Threshold plugin.

Abbreviations: $\mathrm{BA}$, Brodmann area; $\mathrm{CBT}$, cognitive behavior therapy; FDR, false discovery rate.

and facial emoticons in the temporal lobe, fusiform gyrus, and insular cortex compared to the offline-CBT group.

\section{The effect of game-CBT on sociality in ASD adolescents}

Game-CBT for ASD adolescents in this study was effective at improving sociality to a similar degree as offline-CBT. The improvement with regard to emotion recognition, assessed by the percentage of emotional words and facial emoticons identified correctly, was also similar between the game-CBT group and the offline-CBT group. These results are consistent with previous results with CBT using a computer or video game format. ${ }^{1,6,7}$ Interestingly, in our study, the game-CBT group showed an improved social interaction score, a subscale of SCQ, while the offline-CBT group showed an improved social behavior score. Indeed, others have shown that computer games can deliver effective CBT by providing a proper virtual space for practicing social interaction, social cognition training, and sociality education in high-functioning patients with ASD. ${ }^{12,13}$
In this study, we report two novel findings. First, a short intervention (6 weeks) using an online game improved emotion recognition in ASD adolescents. This result builds upon previous studies that utilized a relatively long intervention period of at least 15 weeks. One previous study used Social Cognition and Interaction Training for optimal use with high-functioning autism adults for 18 weeks ${ }^{29}$ and the other implemented 15 weeks of social software..$^{30}$ Both improved emotion recognition in patients with ASD. Second, we used a readymade prosocial game that originally had no intention of improving sociality but was designed simply for leisure. The original online game was used as a platform to conduct the CBT protocol by professional study personnel. To the best of our knowledge, this study is the first trial to compare the effectiveness of the same CBT protocol in the form of game-CBT with a readymade prosocial online game and offline-CBT in adolescents with ASD. Taken together, we cautiously suggest that $\mathrm{CBT}$ using an online game can increase social interaction in a virtual space to improve sociality in ASD adolescents. 


\section{Difference in the changes of brain activity in response to emotional words and facial emoticons between game-CBT and offline-CBT}

The game-CBT group in this study showed different brain changes in response to emotional words and facial emoticons compared to the offline-CBT group. In response to emotional words, the brain activity within the temporal and parietal cortices in the game-CBT group increased, while the brain activity within cingulate and parietal cortices in the offline-CBT group increased. The activation within occipitotemporal areas in response to emotional words is thought to be associated with the lexical meaning of words and the task relevance of visually presented words. ${ }^{31,32}$ The parietal cortex responds to the emotional content of word stimuli. ${ }^{33}$ The cingulate gyrus is thought to be associated with social cognitive functions, including mentalizing, person perception, self-referential judgment, and inferences about others. ${ }^{34,35}$ Specifically, selfreflection consisting of self-referential judgment and inference is a crucial social function for understanding the minds of other people. ${ }^{36}$ The cingulate gyrus is also associated with emotional valence. ${ }^{37}$ The ventral cingulate is involved in error detection and emotional conflict. ${ }^{38}$ The dorsal cingulate gyrus is associated with error and feedback processing. ${ }^{39}$ On the basis of these results, offline-CBT may be particularly useful in augmenting brain use for emotional valence in response to emotional words in ASD adolescents.

In our previous study, patients with ASD were more familiar with emotional words than with facial expressions on emoticons, because emoticons include both verbal parts of a message as well as nonverbal emotional communication. ${ }^{11}$ In addition, decreased brain activity in response to facial emoticons was thought to be associated with emotional salience ${ }^{9}$ or lack of social insight $t^{40}$ in ASD adolescents. After 6 weeks of game-CBT in this study, ASD adolescents showed increased brain activity within the right cingulate gyrus, left medial frontal gyrus, left cerebellum, left fusiform gyrus, left insula, and sublobar area in response to facial emoticons. In a study that encouraged healthy individuals to control emotional valence of associated situations and memories, brain activation within the bilateral cingulate gyrus, medial frontal cortex, and caudate was observed. ${ }^{41}$ The insular cortex is associated with integration of autonomic and visceral information with emotional and motivational functions. ${ }^{42}$ In addition, the insular cortex is involved in integration of conflicting emotional reactions during incongruent stimuli relative to congruent stimuli. ${ }^{43}$ The fusiform gyrus is highly involved in facial recognition. ${ }^{44}$
Interestingly, the brain activity within fusiform gyrus was similar in response to a wide variety of presentation methods, including drawing and photographs. ${ }^{45}$ In this study, the changes in brain activity within the left fusiform gyrus in the game-CBT group were positively correlated with the changes in the correct response rate for emoticons. Taken together, we suggest that game-CBT intervention with multiple facial stimulations presented by an avatar during game play might encourage ASD adolescents to be more emotionally aroused, to better recognize emotions, and to better consider the associated environments in response to emotionally charged faces.

\section{Limitations}

There were several limitations in this research. First, the number of subjects was not large enough to generalize the results to other highly functioning patients with ASD. Second, the improvement in the correct response to emotional words and facial emoticons may be due to repetition effects. Readers should be cautious in interpreting the results. Finally, the efficiency of game-CBT using three professionals can likely be improved, as these treatments can be provided by a single clinician. Future studies should also assess the efficiency of game-CBT relative to standard treatments.

\section{Conclusion}

An online, prosocial game that incorporated a CBT protocol was as effective as an offline-CBT intervention in ASD adolescents. With the evidence of greater improvement of social interaction and increased brain activity within cingulate gyrus, frontal lobe, and insular cortex in response to emoticons, the game-CBT encouraged ASD adolescents to better recognize emotions and more accurately consider associated environments in response to facial emotional stimulation. However, the game-CBT was not as effective at increasing brain use for emotional valence in response to emotional words as the offline counterpart.

\section{Acknowledgments}

The authors convey special thanks to Game Culture Foundation. This work was supported by a Korea Creative Content Agency grant (R2014040055).

\section{Disclosure}

The authors report no conflicts of interest in this work.

\section{References}

1. Allison T, Puce A, McCarthy G. Social perception from visual cues: role of the STS region. Trends Cogn Sci. 2000;4(7):267-278. 
2. Charman T. Why is joint attention a pivotal skill in autism? Philos Trans R Soc Lond B Biol Sci. 2003;358(1430):315-324.

3. Mundy P, Sigman M, Kasari C. A longitudinal study of joint attention and language development in autistic children. J Autism Dev Disord. 1990;20(1):115-128.

4. Hendricks DR, Wehman P. Transition from school to adulthood for youth with autism spectrum disorders. Focus Autism Other Dev Disabl. 2009; 24(2):77-88.

5. Moxon L, Gates D. Children with autism: supporting the transition to adulthood. Educ Child Psychol. 2001;18(2):28-40.

6. Hillier A, Fish T, Cloppert P, Beversdorf DQ. Outcomes of a social and vocational skills support group for adolescents and young adults on autism spectrum. Focus Autism Other Dev Disabl. 2007;22(2): $107-115$

7. Golan O, Baron-Cohen S. Systemizing empathy: teaching adults with Asperger syndrome or high-functioning autism to recognize complex emotions using interactive multimedia. Dev Psychopathol. 2006; 18(2):591-617.

8. Howlin P, Yates P. The potential effectiveness of social skills groups for adults with autism. Autism. 1999;3(3):299-307.

9. Grelotti DJ, Gauthier I, Schultz RT. Social interest and the development of cortical face specialization: what autism teaches us about face processing. Dev Psychobiol. 2002;40(3):213-225.

10. Hauck M, Fein D, Maltby N, Waterhouse L, Feinstein C. Memory for faces in children with autism. Child Neuropsychol. 1998;4(3):187-198.

11. Han DH, Yoo HJ, Kim BN, McMahon W, Renshaw PF. Brain activity of adolescents with high functioning autism in response to emotional words and facial emoticons. PLoS One. 2014;9(3):e91214.

12. Kandalaft MR, Didehbani N, Krawczyk DC, Allen TT, Chapman SB. Virtual reality social cognition training for young adults with highfunctioning autism. J Autism Dev Disord. 2013;43(1):34-44.

13. Crowder SA, Merritte K. The possible therapeutic benefits of utilizing motion gaming systems on pediatric patients presenting autism. Tenn Med. 2013;106(8):41-43.

14. Wallace S, Parsons S, Westbury A, White K, White K, Bailey A. Sense of presence and atypical social judgments in immersive virtual environments. Responses of adolescents with autism spectrum disorders. Autism. 2010;14(3):199-213.

15. Whalen C, Liden L, Ingersoll B, Dallaire E, Liden S. Behavioral improvements associated with computer-assisted instruction for children with developmental disabilities. J Speech Lang Pathol Appl Behav Anal. 2006;1(1):11-26.

16. Parsons S, Mitchell P, Leonard A. Do adolescents with autistic spectrum disorders adhere to social conventions in virtual environments? Autism. 2005;9(1):95-117.

17. Pope AT, Palsson OS. Helping video games 'Rewire Our Minds.' Paper presented at: Playing by the Rules Conference. University of Chicago Cultural Policy Center; October 26-27; 2001; Chicago, IL.

18. Wilkinson N, Ang RP, Goh DH. Online video game therapy for mental health concerns: a review. Int J Soc Psychiatry. 2008;54(4): 370-382.

19. Greitemeyer T, Osswald S. Effects of prosocial video games on prosocial behavior. J Pers Soc Psychol. 2010;98(2):211-221.

20. Gentile DA, Anderson CA, Yukawa S, et al. The effects of prosocial video games on prosocial behaviors: international evidence from correlational, longitudinal, and experimental studies. Pers Soc Psychol Bull. 2009;35(6):752-763.

21. Greitemeyer T, Osswald S, Brauer M. Playing prosocial video games increases empathy and decreases schadenfreude. Emotion. 2010; 10(6):796-802.

22. Suzuki K, Matsuzaki H, Iwata K, et al. Plasma cytokine profiles in subjects with high-functioning autism spectrum disorders. PLoS One. 2011;6(5):e20470.

23. Kim YS, Cheon KA, Kim BN, et al. The reliability and validity of Kiddie-Schedule for Affective Disorders and Schizophrenia-Present and Lifetime Version-Korean version (K-SADS-PL-K). Yonsei Med $J$. 2004;45(1):81-89.
24. Park IJ, Min KH. Making a list of Korean emotion terms and exploring dimensions underlying them. Korean J Soc Pers Psychol. 2005;19(1): 109-129.

25. Berument SK, Rutter M, Lord C, Pickles A, Bailey A. Autism screening questionnaire: diagnostic validity. Br J Psychiatry. 1999;175: 444-451.

26. Yoo HJ. The Social Communication Questionnaire. Seoul, Republic of Korea: Hakjisa Publisher; 2008

27. Yeom TH, Park YS, Oh KJ, Lee YH. Korean version Wechsler adult intelligence scale. Seoul, Republic of Korea: Korean Guidance; 1992.

28. Forman SD, Cohen JD, Fitzgerald M, Eddy WF, Mintun MA, Noll DC. Improved assessment of significant activation in functional magnetic resonance imaging (fMRI): use of a cluster-size threshold. Magn Reson Med. 1995;33(5):636-647.

29. Turner-Brown LM, Perry TD, Dichter GS, Bodfish JW, Penn DL. Brief report: feasibility of social cognition and interaction training for adults with high functioning autism. $J$ Autism Dev Disord. 2008;38(9):1777-1784.

30. Baron-Cohen S, Wheelwright S, Hill J, Raste Y, Plumb I. The "Reading the Mind in the Eyes" test revised version: a study with normal adults, and adults with Asperger syndrome or high-functioning autism. JChild Psychol Psychiatry. 2001;42(2):241-251.

31. Abdullaev YG, Posner MI. Event-related brain potential imaging of semantic encoding during processing single words. Neuroimage. 1998;7(1):1-13.

32. Hinojosa JA, Méndez-Bértolo C, Pozo MA. Looking at emotional words is not the same as reading emotional words: behavioral and neural correlates. Psychophysiology. 2010;47(4):748-757.

33. Carretié L, Hinojosa JA, Albert J, et al. Modulation of ongoing cognitive processes by emotionally intense words. Psychophysiology. 2008; 45(2):188-196.

34. Vogeley K, Bussfeld P, Newen A, et al. Mind reading: neural mechanisms of theory of mind and self-perspective. Neuroimage. 2001; 14(1 Pt 1):170-181.

35. Mitchell JP, Macrae CN, Banaji MR. Dissociable medial prefrontal contributions to judgments of similar and dissimilar others. Neuron. 2006;50(4):655-663.

36. Jenkins AC, Macrae CN, Mitchell JP. Repetition suppression of ventromedial prefrontal activity during judgments of self and others. Proc Natl Acad Sci U S A. 2008;105(11):4507-4512.

37. Lewis PA, Critchley HD, Rotshtein P, Dolan RJ. Neural correlates of processing valence and arousal in affective words. Cereb Cortex. 2007;17(3):742-748.

38. Botvinick M, Nystrom LE, Fissell K, Carter CS, Cohen JD. Conflict monitoring versus selection-for-action in anterior cingulate cortex. Nature. 1999;402(6758):179-181.

39. Polli FE, Barton JJ, Cain MS, Thakkar KN, Rauch SL, Manoach DS. Rostral and dorsal anterior cingulate cortex make dissociable contributions during antisaccade error commission. Proc Natl Acad Sci U S A 2005;102(43):15700-15705.

40. Baron-Cohen S, Leslie AM, Frith U. Does the autistic child have a "theory of mind"? Cognition. 1985;21(1):37-46.

41. Posner J, Russell JA, Gerber A, et al. The neurophysiological bases of emotion: an fMRI study of the affective circumplex using emotiondenoting words. Hum Brain Mapp. 2009;30(3):883-895.

42. Jones CL, Ward J, Critchley HD. The neuropsychological impact of insular cortex lesions. J Neurol Neurosurg Psychiatry. 2010;81(6):611-618.

43. Citron FM, Gray MA, Critchley HD, Weekes BS, Ferstl EC. Emotional valence and arousal affect reading in an interactive way: neuroimaging evidence for an approach-withdrawal framework. Neuropsychologia. 2014;56:79-89.

44. Kanwisher N, McDermott J, Chun MM. The fusiform face area: a module in human extrastriate cortex specialized for face perception. J Neurosci. 1997;17(11):4302-4311.

45. Tong F, Nakayama K, Moscovitch M, Weinrib O, Kanwisher N. Response properties of the human fusiform face area. Cogn Neuropsychol. 2000;17(1):257-280. 


\section{Publish your work in this journal}

Neuropsychiatric Disease and Treatment is an international, peerreviewed journal of clinical therapeutics and pharmacology focusing on concise rapid reporting of clinical or pre-clinical studies on a range of neuropsychiatric and neurological disorders. This journal is indexed on PubMed Central, the 'PsycINFO' database and CAS, and is the official journal of The International Neuropsychiatric Association (INA). The manuscript management system is completely online and includes a very quick and fair peer-review system, which is all easy to use. Visit http://www.dovepress.com/testimonials.php to read real quotes from published authors.

\footnotetext{
Submit your manuscript here: http://www.dovepress.com/neuropsychiatric-disease-and-treatment-journal
} 